\title{
The Post-Surgical Inflammatory Response Provokes Enhanced Tumour Recurrence: A Crucial Role for Neutrophils
}

\author{
M.P. van den Tol $^{a} \quad$ S. ten Raa ${ }^{b}$ W.M.U. van Grevenstein ${ }^{b}$ M.E.E. van Rossen ${ }^{c}$ \\ J. Jeekel ${ }^{b}$ C.H.J.van Eijck ${ }^{b}$ \\ a Department of Surgical Oncology, VU University Medical Centre, Amsterdam, \\ ${ }^{b}$ Department of General Surgery, Erasmus Medical Centre, Rotterdam, and ' Department of General Surgery, \\ Albert Schweitzer Hospital, Dordrecht, The Netherlands
}

\section{Key Words}

Neutrophils • Post-surgical inflammatory response •

Tumour recurrence

\begin{abstract}
Background/Aims: Peritoneal trauma activates a cascade of peritoneal defence mechanisms responsible for postoperative intra-abdominal tumour recurrence. After peritoneal trauma, inflammatory cells and soluble factors are present in the abdominal cavity and can be captured in lavage fluids. The present study evaluated which component enhances intra-abdominal tumour recurrence. Furthermore, we evaluated which inflammatory cells are present and studied the influence of anti-neutrophil serum (ANS) on peritoneal tumour recurrence. Methods: In a peritoneal trauma model in rats, postoperative lavage fluids were collected and separated into cellular and supernatant components. Both components were injected in naïve rats together with CC531s colon carcinoma cells. In a second experiment, rats were treated with one or three doses of ANS. Results: Intraperitoneal injection of naïve recipients with inflammatory cells or supernatant resulted in significant tumour recurrence. Severe peritoneal trauma provoked significant intra-abdominal neutrophil influx which could be prevented by ANS. Treatment with one dose did not affect blood cell counts and significantly reduced tumour recurrence. Treatment with three doses of ANS decreased blood lymphocytes, mono-
\end{abstract}

cytes, and neutrophils and induced tumour load. Conclusions: Neutrophils play a crucial role in postoperative adhesion and growth of spilled tumour cells after surgical peritoneal trauma. Prevention of peritoneal neutrophil influx reduces local tumour recurrence.

Copyright $\odot 2007$ S. Karger AG, Basel

\section{Introduction}

Locoregional tumour recurrence of colorectal carcinomas remains an important complication of potentially curative surgical intra-abdominal interventions. In a clinical study, Busch et al. [1] suggested an association between recurrent tumour disease and extent of surgical injury. It has also become evident from experimental studies $[2,3]$ that enhanced tumour cell adherence and tumour growth are inevitable repercussions of surgical peritoneal trauma. The pathogenesis of the processes responsible for postoperative intra-abdominal tumour recurrence is only partly clarified. In a previous study [4], we demonstrated that within a few hours after infliction of peritoneal trauma, factors in the abdominal cavity could be captured in lavage fluid which enhance tumour recurrence in naïve, non-operated recipients. The inflammatory reaction after surgery is not only responsible for the wound-healing process, but also induces tumour recurrence. During this inflammatory response, perito-

\section{KARGER}

Fax +4161306 1234

E-Mail karger@karger.ch

www.karger.com
(ㄷ) 2007 S. Karger AG, Basel

0253-4886/07/0245-0388\$23.50/0

Accessible online at:

www.karger.com/dsu
C.H.J. van Eijck, $\mathrm{MD}, \mathrm{PhD}$

Department of General Surgery

Erasmus Medical Centre, PO Box 9100

NL-3007 AC Rotterdam (The Netherlands)

Tel. +31 10291 2351, Fax +31 10463 4746, E-Mail sandertenraa@hotmail.com 
neal lymphocytes, submesothelial monocytes, neutrophils, and mesothelial cells act under the control of locally expressed cytokines, chemokines, and adhesion molecules $[5,6]$.

In the present study, we focus our attention on the individual capacity of inflammatory peritoneal cells and soluble factors to ascertain which element is mainly responsible for enhanced tumour recurrence. Secondly, assuming that post-traumatic intra-abdominal influx of neutrophils is an important factor in the dynamic cascade of peritoneal defence, possibly responsible for local tumour recurrence, prevention of polymorphonuclear neutrophil (PMN) influx might influence this process. Therefore, a second experiment was performed to evaluate whether post-traumatic intra-abdominal PMN influx could be reduced by treatment with anti-neutrophil serum (ANS) and, if so, whether this reduction could influence postoperative tumour development.

\section{Materials and Methods}

Animals

Female inbred WAG rats of reproductive age weighing 140 $180 \mathrm{~g}$ were obtained from Harlan-CPB (Austerlitz, The Netherlands). They were bred under specific-pathogen-free conditions, kept in a standard laboratory environment (temperature 20$24^{\circ} \mathrm{C}$, relative humidity $50-60 \%, 12$-hour light/dark cycles), and fed with standard rat food and water ad libitum. The experimental protocol was approved by the Animal Experiments Committee under the national Experiments on Animals Act and adhered to the rules laid down in this national law that serves the implementation of 'Guidelines on the Protection of Experimental Animals' by the Council of Europe (1986), Directive 86/609/EC.

\section{Tumour}

The tumour CC531s is a moderately differentiated, weakly immunogenic colonic adenocarcinoma induced in WAG rats by 1,2dimethylhydrazine [7]. It is transplantable in syngeneic WAG rats. The tumour is maintained as a cell culture in RPMI 1640 medium (RPMI) supplemented with $5 \%$ fetal calf serum (virus and Mycoplasma screened), 1\% penicillin (5,000 U/ml), 1\% streptomycin $(5,000 \mathrm{U} / \mathrm{ml})$, and $1 \% \mathrm{~L}$-glutamine $(200 \mathrm{mmol})$. RPMI and all supplements were obtained from Life Technologicies (Bre$\mathrm{da}$, The Netherlands). The cells were passaged once a week using trypsin $(0.05 \%)$ and EDTA $(0.02 \%)$. Before use in vivo, the tumour cells were harvested from stationary cultures by gentle trypsinization $\left(5 \mathrm{~min}, 37^{\circ} \mathrm{C}\right)$, centrifugation $(5 \mathrm{~min}, 700 \mathrm{~g}$ ), and resuspension in RPMI, providing cell suspensions with a viability $>90 \%$. Tumour CC531s is relatively insensitive to chemotherapy, but is sensitive to the effects of biological response modifiers.

Gathering of Lavage Fluid for Passive Transfer Experiment

Under isoflurane anaesthesia, in 14 rats a laparotomy was performed; exposure and rubbing of the uterine horns and a $5-\mathrm{cm}$ part of the small intestine with surgical Medipress gauze inflicted subsequent trauma to the peritoneum [4]. Rubbing was performed with a device enabling the application of a constant pressure of $120 \mathrm{~g} / \mathrm{m}^{2}$. In this way, a standardized degree of peritoneal trauma can be inflicted. The abdomen was closed in one layer with silk 2- 0 sutures. After $5 \mathrm{~h}$, a second laparotomy was performed, during which the abdominal cavity was lavaged with $5 \mathrm{ml} \mathrm{RPMI}$. After massaging the abdomen, the remaining fluid was aspirated, pooled, and kept on ice until further processing.

\section{Experimental Design of Passive Transfer (Experiment 1)}

The collected post-trauma lavage fluid was centrifuged, and the cell pellet was resuspended to the original volume with RPMI and thus divided into a 'supernatant' containing soluble components produced after surgical trauma and a 'cellular' component containing the different cell types present in the abdominal cavity after surgical trauma.

Subsequently, 24 rats were divided into three groups: one group served as control group receiving RPMI; the second group was acceptor for the supernatant of the post-trauma lavage fluid, and the third group was acceptor for the cellular component. Of all three components, $3 \mathrm{ml}$ was injected intraperitoneally together with 0.5 million CC531s cells (in $0.5 \mathrm{ml}$ RPMI) without opening the abdominal cavity. In this way, the factors contained in the different components represented the mediators after surgical abdominal trauma, without inflicting additional trauma (fig. 1).

\section{Rabbit Anti-Rat Neutrophil Serum}

Polyclonal rabbit ANS was purchased from Accurate Chemical \& Scientific (Westbury, N.Y., USA). ANS can deplete blood neutrophils in rats by $99.9 \%$ when administered intraperitoneally at a daily dose of $2 \mathrm{ml} / \mathrm{kg}[8,9]$. The number of blood neutrophils remains at this low level, until the administration of ANS is stopped. At this dose, ANS is not specific for neutrophils only, because the numbers of blood monocytes, lymphocytes, and, to a lesser extent, platelets decrease as well, i.e., by 100,80 , and $25 \%$, respectively [9]. Therefore, a pilot experiment was performed to achieve a dose of ANS at which blood cell counts hardly change, as shown in experiment 2 .

\section{Effect of ANS Treatment on Cell Content in Peritoneal Cavity} and Blood (Experiment 2)

To investigate the influence of ANS treatment on the intra-abdominal neutrophil count and on the rat immune system, the following procedures were performed. Under isoflurane anaesthesia, 65 rats underwent a laparotomy. In 5 rats no peritoneal trauma was inflicted. In 20 rats, standardized severe peritoneal trauma was inflicted without treatment. In 20 rats, severe peritoneal trauma was inflicted (on day 0 ) in rats treated with three intraperitoneal doses of ANS $(1 \mathrm{ml} / \mathrm{kg})$ on days $-1,0$, and +1 . In 20 rats severe peritoneal trauma was inflicted after a single intraperitoneal injection of ANS on day -1. After 5, 72, 96, and $144 \mathrm{~h}, 5$ rats of each group were operated for the second time. During this second laparotomy, the abdominal cavity was lavaged with $5 \mathrm{ml} \mathrm{RPMI}$. After massaging the abdomen, the remaining fluid was aspirated and individually kept on ice until further processing. Blood samples were obtained as well, by cardiac puncture.

The collected lavage fluid samples were separated in a supernatant component and a cellular component by centrifugation (1,500 rpm, $5 \mathrm{~min})$. The cellular component was resuspended in RPMI, the total cell amount was determined, and haematoxylin- 


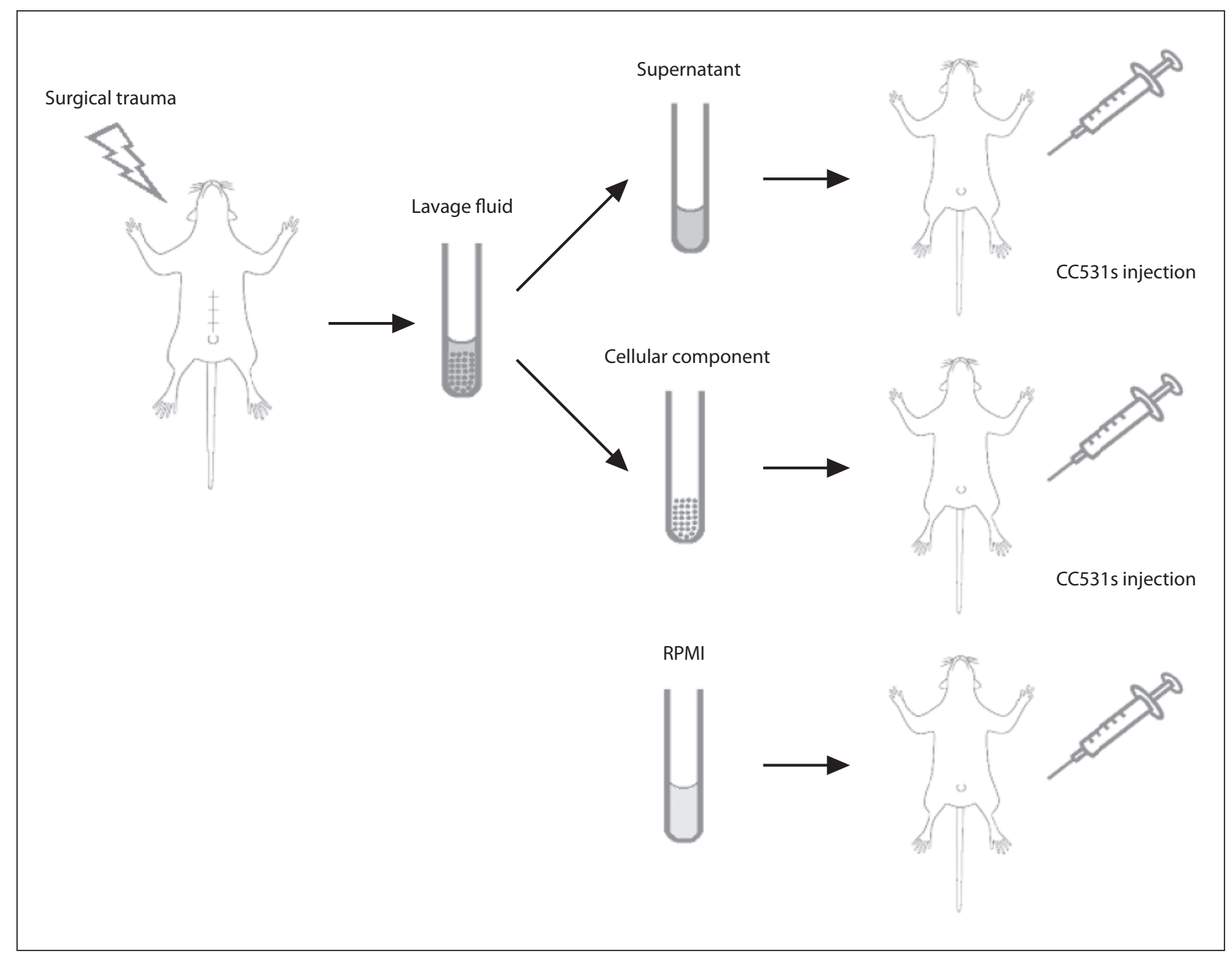

Fig. 1. Experimental design of the passive transfer experiment.

and-eosin-stained cytocentrifuge slides were made for cell differentiation. At $\times 100,100$ cells were counted in duplicate and classified into granulocytes (neutrophils, eosinophils, basophils, and mast cells) and lymphoid cells (mononuclear phagocytes and lymphocytes). Total blood leucocyte counts were determined with a micro cell counter, and duplicate differential counts were carried out on May-Grünwald- and Giemsa-stained blood smears.

\section{Intraperitoneal Tumour Cell Adhesion and Growth after}

Treatment with ANS (Experiments 3 and 4)

A reproducible animal experimental model was used [4]. Briefly, under isoflurane anaesthesia and aseptic conditions, a laparotomy was performed by a midline incision of $5 \mathrm{~cm}$. Both uterine horns were exposed, not touched or rubbed with surgical Medipress gauze, and sutured to the lateral peritoneum both proximally and distally using Surgilene 6-0 sutures. In this way, a standardized degree of peritoneal trauma was inflicted. To in- duce peritoneal metastases, $0.5 \times 10^{6}$ CC531s tumour cells, in $1 \mathrm{ml}$ RPMI, were injected intraperitoneally before closing the abdomen. The abdomen was closed in two layers with 5-0 polyglycolic acid and 2-0 silk sutures.

In experiment 3,9 rats underwent severe peritoneal trauma without treatment. Nine rats underwent severe peritoneal traumas which were treated with an intraperitoneal injection of ANS on day -1 . Before closing the abdomen $0.5 \times 10^{6}$ CC531s tumour cells, in $1 \mathrm{ml}$ RPMI, were injected intraperitoneally in all rats.

In experiment 4 , similar groups were formed, with the difference that the treated group received intraperitoneal ANS injections on days $-1,0$, and +1 . Again, before closing the abdomen, $0.5 \times 10^{6}$ CC531s tumour cells were injected into the abdominal cavity.

\section{Evaluation of Tumour Load}

Three weeks after injection of CC531s tumour cells, all rats were sacrificed, and the intraperitoneal tumour load was scored semi- 


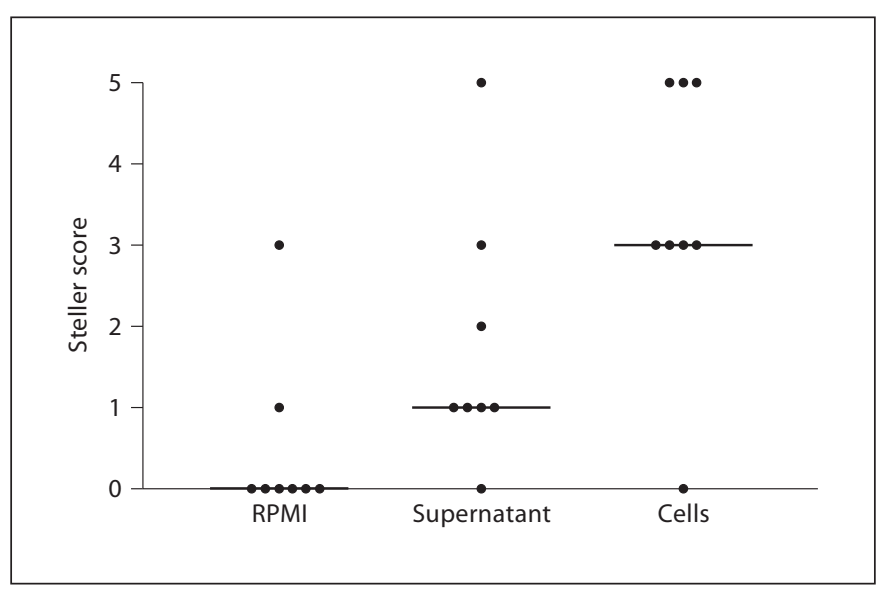

Fig. 2. Median intraperitoneal tumour load and range after passive transfer of RPMI only, the supernatant, or the cellular fraction of lavage fluid collected after infliction of severe peritoneal trauma. RPMI vs. cells: $\mathrm{p}<0.01$; RPMI vs. supernatant: $\mathrm{p}<0.05$; supernatant vs. cells: $\mathrm{p}<0.05$

quantitatively at the following peritoneal sites: right uterine horn, left uterine horn, subcutaneously (at the site of the operative scar), parietal peritoneum (on the lateral abdominal wall sides, where no uterine horns were fixed), kidney, liver, retroperitoneum, and omentum. Scoring was performed by two blinded observers, using a tumour scoring system derived from the peritoneal cancer index (Steller score) $[4,10]$, ranging from 0 to 5 per abdominal site. For each rat, the score at all peritoneal sites was summarized, from which a mean total tumour load per rat could be estimated.

\section{Statistics}

Statistical analysis was performed using one-way ANOVA tests to determine overall differences. If an ANOVA test was significant at the $5 \%$ level, the Newman-Keuls post hoc test was carried out to make a comparison between groups. Data are expressed as mean \pm SEM. For tumour load, median and range were calculated, and statistical analysis was performed using the non-parametric Kruskal-Wallis analysis of variance to determine overall differences, followed by the non-parametric Mann-Whitney $U$ test to compare differences between groups. Statistical significance was defined as $\mathrm{p}<0.05$.

\section{Results}

\section{Effect of Post-Traumatic Lavage Fluid on Peritoneal} Tumour Load

After intraperitoneal injection of the lavage fluid samples collected after surgical trauma, a diffuse peritoneal

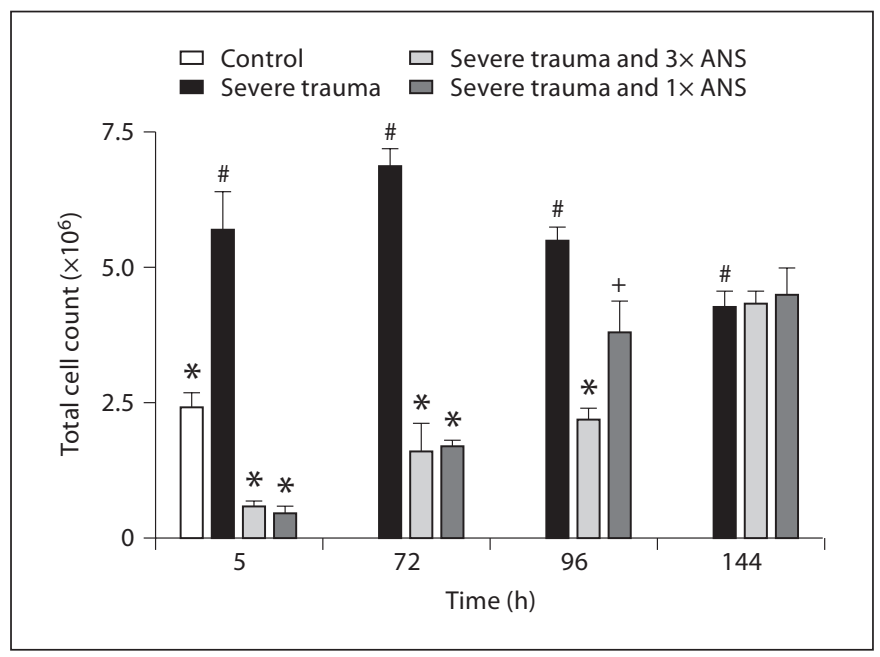

Fig. 3. Mean total cell counts ( \pm SEM) in abdominal lavage fluids after no touch, after infliction of severe peritoneal trauma, or after infliction of severe peritoneal trauma in rats treated with three intraperitoneal doses or one intraperitoneal dose of ANS. Fluids were collected $5,72,96$, and 144 h postoperatively. ${ }^{*} \mathrm{p}<0.001$ and ${ }^{+} \mathrm{p}<0.01$ vs. severe peritoneal trauma; ${ }^{\#} \mathrm{p}<0.05$ vs. no touch.

tumour load was found in all groups (fig. 2). When compared with the control group (RPMI), the cellular factors caused significantly enhanced tumour recurrence $(\mathrm{p}<$ $0.01)$, as well as the supernatant $(\mathrm{p}<0.05)$. Injection of tumour cells with RPMI alone resulted in a median total Steller score of 0 (range $0-3$ ), whereas injection with the supernatant or cells resulted in Steller scores of 1 (range $0-5$ ) and 3 (range 0-5), respectively. Injection with the cellular component of the lavage fluid also resulted in a significantly higher tumour load as compared with the supernatant $(\mathrm{p}<0.05)$.

\section{Cell Content in Peritoneal Cavity and Blood after \\ Surgery and the Effect of ANS Treatment}

There was a significant increase in total intra-abdominal cell count after infliction of severe peritoneal trauma up to $144 \mathrm{~h}$ after the operation (fig. 3). Figure 3 also shows that intraperitoneal administration of three doses of ANS as well as one dose of ANS significantly decreased the total intra-abdominal cell count after infliction of severe peritoneal trauma for at least $96 \mathrm{~h}$ postoperatively $(\mathrm{p}<$ 0.01 ). After treatment with one dose of ANS, the total cell count increased earlier than after treatment with three doses, the differences being significant at $96 \mathrm{~h}(\mathrm{p}<0.01)$ (fig. 3). A marked increase of the percentage of neutrophils was seen in the severely traumatized group $(\mathrm{p}<$ 


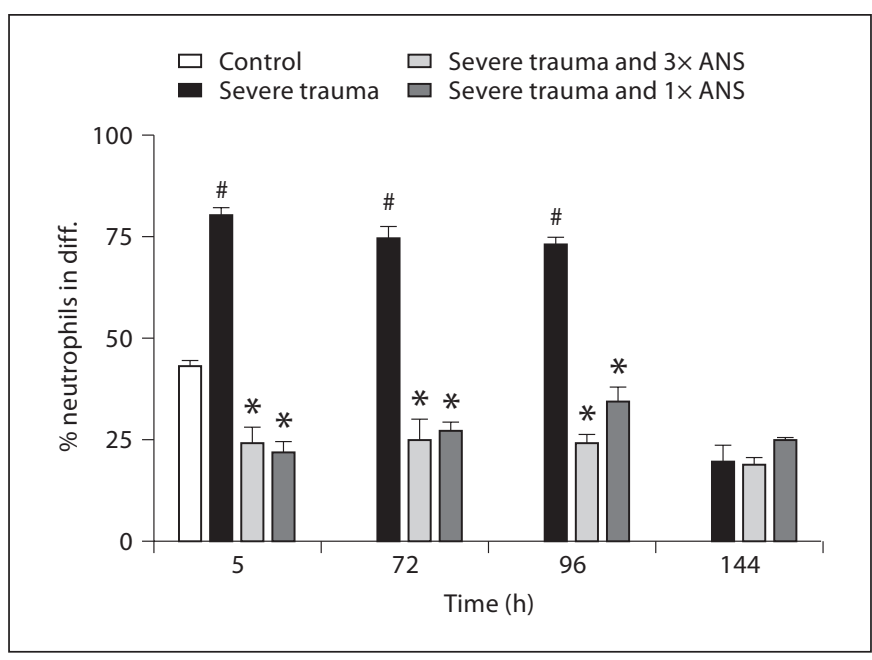

Fig. 4. Mean percentage of neutrophils ( \pm SEM) in abdominal lavage fluids taken from abdominal cavities after no touch, after infliction of severe peritoneal trauma, or after infliction of severe peritoneal trauma in rats treated with three intraperitoneal doses or one intraperitoneal dose of ANS. Fluids were collected 5, 72, 96 , and $144 \mathrm{~h}$ postoperatively. ${ }^{*} \mathrm{p}<0.001$ vs. severe peritoneal trauma; ${ }^{*} \mathrm{p}<0.05$ vs. no touch.

0.01 ). This increase was seen till $96 \mathrm{~h}$ postoperatively (fig. 4). Treating the rats with three doses as well as one dose of ANS did annul this increase (fig. 4).

Figure 5 shows the results of blood differential cell counts in the four groups at different time points. Similar results were found with lymphocyte, monocyte, or neutrophil counts. Treatment with three doses of ANS significantly decreased these cell counts for a period of at least $96 \mathrm{~h}(\mathrm{p}<0.01)$. This effect was not seen after treatment with one dose of ANS.

\section{Intraperitoneal Tumour Development after Treatment} with ANS

As shown in figure 6, a marked tumour load is seen after severe trauma. Treatment with a single dose of ANS significantly reduced the median tumour load in severely traumatized rats $(\mathrm{p}<0.01)$. Surprisingly, intraperitoneal injection of three doses of ANS induced even more tumour load $(\mathrm{p}<0.001)$.

\section{Discussion}

In this study, a cell-seeding model was used to mimic the clinical situation of tumour cell spill during tumour resection.

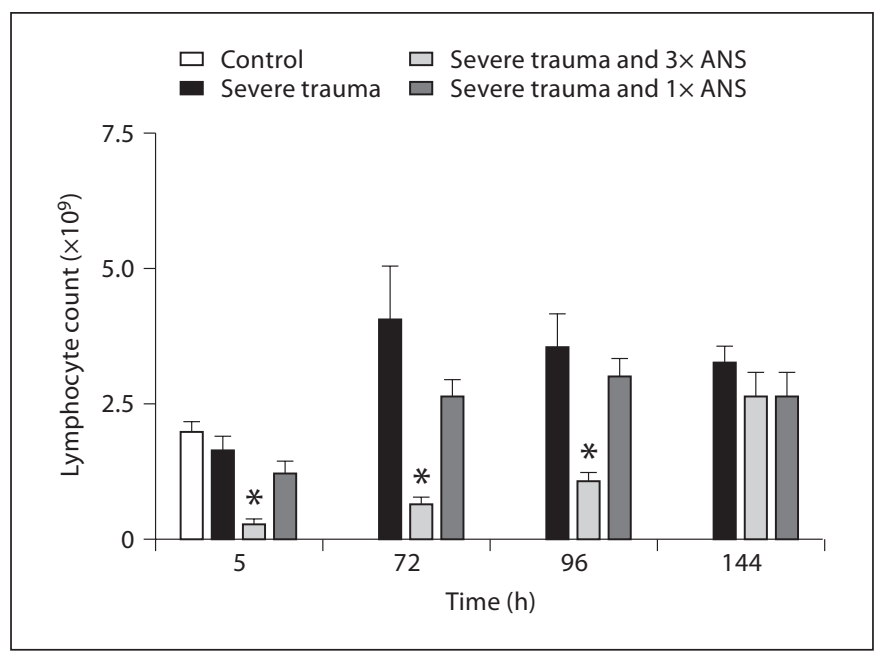

Fig. 5. Mean blood lymphocyte count ( \pm SEM) after no touch, after infliction of severe peritoneal trauma, or after infliction of severe peritoneal trauma in rats treated with three intraperitoneal doses or one intraperitoneal dose of ANS. Blood samples were obtained $5,72,96$, and 144 h postoperatively. ${ }^{*} \mathrm{p}<0.01$ vs. severe peritoneal trauma.

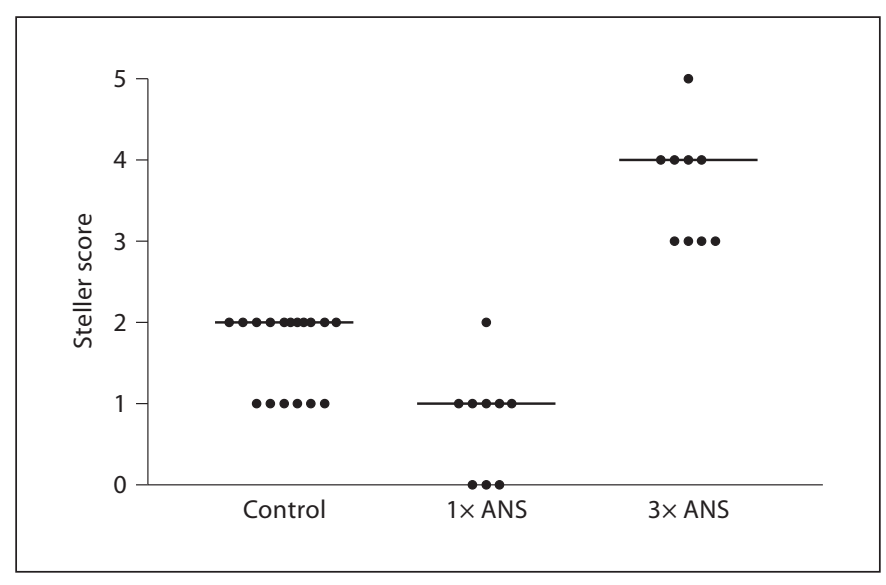

Fig. 6. Median intraperitoneal tumour load and range of severely traumatized rats (control), severely traumatized rats treated with ANS on day -1 ( $1 \times$ ANS), and severely traumatized rats treated with ANS on days $-1,0$, and +1 ( $3 \times$ ANS). Control vs. $1 \times$ ANS: $\mathrm{p}<0.01$; control vs. $3 \times$ ANS: $\mathrm{p}<0.001$.

Following peritoneal trauma, a variety of cytokines, growth factors, and other inflammatory mediators are produced by activated mesothelial cells and by stampeded inflammatory cells [11]. The production of mesothelial cell and inflammatory cell derived chemokines will 
cause an early posttraumatic migration of PMNs and monocytes to the injured peritoneal cavity, in order to promote the peritoneal healing process [12]. However, these mediators and recruited cells not only serve peritoneal healing, but, as shown in a previous study [4], could enhance tumour recurrence in naïve recipients as well. The current study demonstrates that the cellular components of the lavage fluid collected after inflicting surgical trauma, i.e., inflammatory cells, lead to enhanced tumour recurrence. More detailed analysis of the cellular fraction of these lavage fluids revealed a trauma-related influx of PMNs into the abdominal cavity (fig. 4). PMNs use both oxygen-dependent and oxygen-independent processes in killing micro-organisms, but these processes also (further) damage surrounding host tissue $[13,14]$. In vitro, adhesion of activated PMNs to a mesothelial monolayer has been shown to induce retraction, gap formation, and detachment, ending with substantial mesothelial cell injury and exposure of extracellular matrix components, thereby already creating a preferential site for adhesion of free tumour cells [15]. A relation has been demonstrated between extent of tissue trauma and tumour recurrence. In order to diminish tissue trauma and tumour recurrence, minimally invasive surgery is promoted and proved promising in rat models $[4,16]$. In addition, laparoscopic surgery appears to have less impact on the cellular components of the immune response than laparotomy [3]. A recent meta-analysis [17] reported faster postoperative recovery with laparoscopic surgery for colorectal cancer as compared with open surgery, though in this metaanalysis no differences were seen in recurrence rates. However, only a few studies published long-term results with a favourable trend towards laparoscopy [18].
The coincidence of post-traumatic intra-abdominal PMN influx with tumour cell adhesion and growth, however, is no solid proof for the role of PMNs in these pathogenetic processes. Effective inhibition of tissue injury by PMNs has been achieved [19, 20]. Dovi et al. [21] even showed that wound healing was accelerated in PMN-depleted mice. However, whether inhibition of PMNs affected tumour recurrence has not been investigated. The present study demonstrated that a single intraperitoneal dose of ANS could prevent the influx of PMNs without influencing the blood cell count. This study showed that a selective reduction of post-traumatic PMN influx, without causing systemic immunosuppression, was possible and could indeed significantly lower tumour adhesion and growth. Averting the post-traumatic intra-abdominal PMN influx by intraperitoneal injection of three doses of ANS significantly increased tumour recurrence, at first to our surprise. However, treatment with three doses of ANS also significantly decreased blood lymphocyte, monocyte, and PMN counts, thereby seriously compromising the rat immune system. It is conceivable that this immunosuppression promotes tumour growth. We observed earlier that immunosuppression leads to enhanced growth of the tumour used in the current experiments [22].

In conclusion, these studies demonstrated that the early inflammatory sequelae after surgery promote tumour recurrence and that this effect is mainly based on the cellular component of the inflammatory process. Preventing the postoperative influx of PMNs without affecting the systemic immune response reduced peritoneal tumour recurrence.

\section{References}

1 Busch OR, Hop WC, Hoynck van Papendrecht MA, Marquet RL, Jeekel J: Blood transfusions and prognosis in colorectal cancer. $\mathrm{N}$ Engl J Med 1993;328:1372-1376.

$\checkmark 2$ Raa ST, Oosterling SJ, van der Kaaij NP, van den Tol MP, Beelen RH, Meijer S, van Eijck $\mathrm{CH}$, van der Sijp JR, van Egmond M, Jeekel J: Surgery promotes implantation of disseminated tumor cells, but does not increase growth of tumor cell clusters. J Surg Oncol 2005;92:124-129.

>3 Jacobi CA, Ordemann J, Zieren HU, Volk HD, Bauhofer A, Halle E, Müller JM: Increased systemic inflammation after laparotomy vs. laparoscopy in an animal model of peritonitis. Arch Surg 1998;133:258-262.
4 van den Tol PM, van Rossen EE, van Eijck $\mathrm{CH}$, Bonthuis F, Marquet RL, Jeekel H: Reduction of peritoneal trauma by using nonsurgical gauze leads to less implantation metastasis of spilled tumor cells. Ann Surg 1998;227:242-248.

5 Ellis H, Harrison W, Hugh TB: The healing of peritoneum under normal and pathological conditions. Br J Surg 1965;52:471-476.

6 Topley N, Liberek T, Davenport A, Li FK, Fear H, Williams JD: Activation of inflammation and leukocyte recruitment into the peritoneal cavity. Kidney Int 1996;56 (suppl):S17-S21.
7 Marquet RL, Westbroek DL, Jeekel J: Interferon treatment of a transplantable rat colon adenocarcinoma: importance of tumor site. Int J Cancer 1984;33:689-692.

-8 Sandler H, Hogstorp H, Lundberg C, Gerdin B: Antiserum-induced neutropenia in the rat: characterization of a rabbit anti-rat neutrophil serum. Br J Exp Pathol 1987;68:7180.

$\checkmark 9$ de Vree WJ, Essers MC, de Bruijn HS, Star WM, Koster JF, Sluiter W: Evidence for an important role of neutrophils in the efficacy of photodynamic therapy in vivo. Cancer Res 1996;56:2908-2911. 
10 Steller EP, Ottow RT, Eggermont AM, Marquet RL, Sugarbaker PH: Local conditions in the host influence immunotherapy with interleukin-2 and LAK cells. Cancer Detect Prev 1988;12:81-90.

11 Holmdahl L, Ivarsson ML: The role of cytokines, coagulation, and fibrinolysis in peritoneal tissue repair. Eur J Surg 1999;165: 1012-1019.

$\checkmark 12$ Novitsky YW, Litwin DE, Callery MP: The net immunologic advantage of laparoscopic surgery. Surg Endosc 2004;18:1411-1419.

13 Weiss SJ: Tissue destruction by neutrophils. N Engl J Med 1989;320:365-376.

14 Martin P, Leibovich SJ: Inflammatory cells during wound repair: the good, the bad and the ugly. Trends Cell Biol 2005;15:599-607.
15 Andreoli SP, Mallett C, Williams K, McAteer JA, Rothlein R, Doerschuk CM: Mechanisms of polymorphonuclear leukocyte mediated peritoneal mesothelial cell injury. Kidney Int 1994;46:1100-1109.

16 Bouvy ND, Marquet RL, Jeekel H, Bonjer HJ: Impact of gas(less) laparoscopy and laparotomy on peritoneal tumor growth and abdominal wall metastases. Ann Surg 1996; 224:694-700; discussion 700-701.

17 Reza MM, Blasco JA, Andradas E, Cantero $\mathrm{R}$, Mayol J: Systematic review of laparoscopic versus open surgery for colorectal cancer. Br J Surg 2006;93:921-928.

18 Lacy AM, Garcia-Valdecasas JC, Delgado S, Castells A, Taura P, Pique JM, Visa J: Laparoscopy-assisted colectomy versus open colectomy for treatment of non-metastatic colon cancer: a randomised trial. Lancet 2002; 359:2224-2229.
19 Zagorski J, Wahl SM: Inhibition of acute peritoneal inflammation in rats by a cytokine-induced neutrophil chemoattractant receptor antagonist. J Immunol 1997;159: 1059-1062.

20 Wang P, Chen H, Qin H, Sankarapandi S, Becher MW, Wong PC, Zweier JL: Overexpression of human copper, zinc-superoxide dismutase (SOD1) prevents postischemic injury. Proc Natl Acad Sci USA 1998;95:45564560.

21 Dovi JV, He LK, DiPietro LA: Accelerated wound closure in neutrophil-depleted mice. J Leukoc Biol 2003;73:448-455.

22 Van de Vrie W, Marquet RL, Eggermont AM: Cyclosporin A enhances locoregional metastasis of the CC531 rat colon tumour. J Cancer Res Clin Oncol 1997;123:21-24. van den Tol/ten Raa/van Grevenstein/ van Rossen/Jeekel/van Eijck 